\title{
Dislipidemia em crianças e adolescentes de diferentes faixas etárias residentes no
}

\section{município de Goiânia}

\author{
Dyslipidemia in children and teenager of different age groups living in the city of Goiânia \\ Dislipidemia en niños y adolescentes de diferentes grupos de edad residentes en la ciudad de \\ Goiânia
}

\section{Resumo}

Objetivo: Investigar a presença de dislipidemias e o estado nutricional em um grupo de pacientes, de zero a doze anos, residentes no município de Goiânia. Metodologia: Estudo analítico do tipo série de casos, no qual foram analisados 130 prontuários eletrônicos de pacientes de 0-12 anos residentes em Goiânia Os participantes foram divididos em quatro grupos de diferentes faixas etárias: o grupo 1 são crianças de zero a três anos, o grupo 2 de quatro a seis, o grupo 3 de sete a nove e o grupo 4 de dez a doze anos. Os dados coletados foram: gênero, idade, peso, altura e exames laboratoriais alterados. O Score Z foi calculado no softwear profissional Cookie ${ }^{\circledR}$ e segue as recomendações do SISVAN/DATASUS (Vigilância Alimentar e Nutricional) que são baseados em critérios da OMS. E a dislipidemia foi classificada de acordo com a elevação do perfil lipídico. Os dados obtidos foram dispostos em planilhas no Microsoft Excel® (versão Office 2007) e para o processamento dos resultados utilizou-se estatística descritiva. Resultados: No grupo 1 foram analisados 40 prontuários e observou-se que $40 \%$ das crianças apresentavam dislipidemia e 5\% eram obesas, a associação de dislipidemia e obesidade foi de $0 \%$. Dos 30 prontuários analisados no grupo $220 \%$ apresentavam dislipidemia e 17\% eram obesos, 6,7\% eram obesos e dislipêmicos. No grupo 3 foram analisadas 30 crianças, $40 \%$ apresentavam dislipidemia e $23 \%$ obesidade, $6,7 \%$ eram obesos e dislipêmicos. Já o grupo 4, após avaliação de 30 prontuários observou-se que 30\% dos adolescentes apresentavam dislipidemia e $60 \%$ obesidade, a associação de dislipêmicos e obesos foi 13,3\%. Conclusão: Foi observado um número significativo de crianças e adolescentes residentes em Goiânia que apresentam dislipidemia e obesidade, porém o fato de as crianças terem apresentado obesidade não necessariamente associa-se com o quadro de dislipidemia. Tais dados são importantes para elaboração de políticas de saúde pública que podem auxiliar em estatísticas do SISVAN/DATASUS. Dessa forma incentivar a população goiana e outras comunidades a cuidar da sua alimentação. A melhor forma de evitar e tratar essas doenças na fase infantil é a dietoterapia e regularização da pratica de atividades físicas.

Palavras-chave: Adolescentes; Crianças; Dislipidemia.

\footnotetext{
Abstract

Objective: To investigate the presence of dyslipidemia and nutritional status in a group of patients, aged from zero to twelve years, living in the city of Goiânia. Methodology: Analytical case series study, in which 130 electronic records
} 
of patients aged 0-12 years living in Goiânia were analyzed. Participants were divided into four groups of different age groups: group 1 are children aged zero to three years, group 2 from four to six, group 3 from seven to nine and group 4 from ten to twelve years old. The data collected were: gender, age, weight, height and altered laboratory tests. The Z-Score was calculated in Cookie ${ }^{\circledR}$ professional softwear and follows the recommendations of SISVAN/DATASUS (Food and Nutritional Surveillance) which are based on WHO criteria. And dyslipidemia was classified according to the elevation of the lipid profile. The data obtained were arranged in spreadsheets in Microsoft Excel ${ }^{\circledR}$ (Office 2007 version) and descriptive statistics were used to process the results. Results: In group 1, 40 medical records were analyzed and it was observed that $40 \%$ of the children had dyslipidemia and $5 \%$ were obese, the association of dyslipidemia and obesity was $0 \%$. Of the 30 medical records analyzed in group 2, 20\% had dyslipidemia and $17 \%$ were obese, $6.7 \%$ were obese and dyslipemic. In group 3, 30 children were analyzed, $40 \%$ had dyslipidemia and $23 \%$ obesity, $6.7 \%$ were obese and dyslipidemic. In group 4, after evaluating 30 medical records, it was observed that $30 \%$ of the adolescents had dyslipidemia and $60 \%$ were obese, the association between dyslipidemic and obese was $13.3 \%$. Conclusion: A significant number of children and adolescents living in Goiânia were found to have dyslipidemia and obesity, but the fact that children were obese is not necessarily associated with dyslipidemia. Such data are important for the elaboration of public health policies that can help in SISVAN/DATASUS statistics. In this way, encourage the population of Goiás and other communities to take care of their food. The best way to prevent and treat these diseases in childhood is diet therapy and regularization of physical activity.

Keywords: Adolescents; Kids; Dyslipidemia.

\begin{abstract}
Resumen
Objetivo: Investigar la presencia de dislipidemia y estado nutricional en un grupo de pacientes de cero a doce años residentes en la ciudad de Goiânia. Metodología: Estudio analítico de series de casos, en el que se analizaron 130 registros electrónicos de pacientes de 0-12 años residentes en Goiânia. Los participantes se dividieron en cuatro grupos de diferentes grupos de edad: grupo 1 son niños de cero a tres años, grupo 2 a partir de cuatro. a seis, grupo 3 de siete a nueve y grupo $4 \mathrm{de}$ diez a doce años. Los datos recogidos fueron: sexo, edad, peso, talla y pruebas de laboratorio alteradas. El Z-Score se calculó en el software profesional Cookie ${ }^{\circledR}$ y sigue las recomendaciones de SISVAN / DATASUS (Vigilancia Alimentaria y Nutricional) que se basan en los criterios de la OMS. Y la dislipidemia se clasificó según la elevación del perfil lipídico. Los datos obtenidos se ordenaron en hojas de cálculo en Microsoft Excel® (versión Office 2007) y se utilizó estadística descriptiva para procesar los resultados. Resultados: En el grupo 1 se analizaron 40 historias clínicas y se observó que el $40 \%$ de los niños presentaba dislipidemia y el 5\% obesidad, la asociación de dislipidemia y obesidad fue de $0 \%$. De las 30 historias clínicas analizadas en el grupo 2, el $20 \%$ presentaba dislipidemia y el $17 \%$ obesidad, el 6,7\% obesidad y dislipémica. En el grupo 3 se analizaron 30 niños, el $40 \%$ tenía dislipidemia y el $23 \%$ obesidad, el 6,7\% eran obesos y dislipidémicos. En el grupo 4, luego de evaluar 30 historias clínicas, se observó que el 30\% de los adolescentes tenían dislipidemia y el $60 \%$ eran obesos, la asociación entre dislipidémicos y obesos fue de 13,3\%. Conclusión: Se encontró que un número significativo de niños y adolescentes que viven en Goiânia tienen dislipidemia y obesidad, pero el hecho de que los niños sean obesos no se asocia necesariamente con dislipidemia. Dichos datos son importantes para la elaboración de políticas de salud pública que puedan ayudar en las estadísticas SISVAN / DATASUS. De esta forma, incentivar a la población de Goiás y otras comunidades a cuidar su alimentación. La mejor forma de prevenir y tratar estas enfermedades en la infancia es la dietoterapia y la regularización de la actividad física.
\end{abstract}

Palabras clave: Adolescentes; Niños; Dislipidemia.

\title{
1. Introdução
}

A dislipidemia é caracterizada por alterações nas concentrações de um ou mais lípides/lipoproteínas encontradas no sangue, são elas: triglicerídeos, colesterol total, lipoproteínas de alta densidade (HDL), lipoproteínas de baixa densidade (LDL) e lipoproteína de muito baixa densidade (VLDL). Estas alterações estão relacionadas com o risco de desenvolvimento de doença coronariana (Fernandes et al., 2011). As causas primárias normalmente estão associadas afatores genéticos e ambientais, como: alimentação rica em açúcares e/ou gorduras, industrializados altamente processados, falta da prática de atividade física e estilo de vida, como o etilismo, tabagismo, etc (Santos et al., 1999). Existem também as causas secundárias que se associam a doenças, como hipotireoidismo, hepatopatias, doenças autoimunes e pós transplantados(Avezum et al., 2015).

As dislipidemias podem ser classificadas de acordo com os resultados laboratoriais em hipercolesterolemia isolada, quando apresentam níveis elevados de LDL colesterol; hipertrigliceridemia isolada, ou quando apresentam o nível de 
triglicerídeo alterado ou com aumento de VLDL associado; e hiperlipemia mista, que consiste nos níveis de LDL e triglicerídeo elevados e HDL-C baixo (Avezum et al., 2015).

Um programa estadunidense, o Programa Nacional de Educação sobre Colesterol (NCEP), em 2001, afirmou que níveis de lipídios elevados no sangue são um dos precursores da arterosclerose coronariana, que é um problema de saúde pública no Brasil. já que é tardiamente diagnosticado e quando agrava leva a doenças coronarianas. O que mais preocupa é o fato de que antes os problemas associados a sobrepeso afetavam principalmente a população adulta, porém atualmente atingem crianças e adolescentes (De Franca et al., 2006).

Em crianças, o mais comum é a elevação do colesterol total isolado ou a associação de níveis altos de LDL e em menor número hipertrigliceridemia (Cook et al., 2011). Nestas, a hipercolesterolemia associada a obesidade aumenta os riscos de doenças coronarianas, diabetes melittus e até aterosclerose infantil. Esses quadros mostram grande piora quando a criança não apresenta uma alimentação saudável e não pratica atividade física. A progressão dessa doença para a fase adulta é muito comum, além disso, o tabagismo, alcoolismo e a falta de controle alimentar vem como fatores intensificadores para piora do quadro (Mello et al., 2004; Avezum et al., 2015). O trabalho realizado por Hafe, em 2015, mostra que obesidade na infância prediz a obesidade na fase adulta, e quando associados aumento das medidas antropométricas, principalmente na região abdominal, prejudica o tratamento de diabetes e assim gera resistência insulínica levando a um quadro de hiperinsulinemia.

O excesso de peso pode ser mensurado a partir de várias técnicas, como a antropometria, que discorre da relação cintura quadril, índice de massa corporal (IMC), entre outras. O cálculo normalmente utiliza dados como peso e altura, onde valores entre $25,0 \mathrm{~kg} / \mathrm{m} 2$ e $29,9 \mathrm{~kg} / \mathrm{m} 2$ compreendem sobrepeso e valores de $\mathrm{IMC} \geq 30,0 \mathrm{~kg} / \mathrm{m} 2$ correspondem a obesidade (Brasil, 2004). Dados da Organização Mundial da Saúde (OMS) mostram que IMC maior que $21 \mathrm{~kg} / \mathrm{m}^{2}$, ou seja, parte de pacientes eutróficos, os que tem sobrepeso e os que tem obesos, podem sugerir aproximadamente $58 \%$ dos casos de diabetes, $21 \%$ de doenças isquêmicas do coração e $8 \%$ a $42 \%$ de certos tipos de câncer (Dos Anjos, 2006). O score Z utiliza a avaliação antropométrica que é composta pelas medidas de peso, altura, gênero, idade e pregas. Nos casos de crianças a avaliação é feita sem as pregas pois não é uma pratica dos nutricionistas, em adultos as pregas e medidas são utilizadas.

O Ministério da Saúde afirmou, em 2016, que 56,9\% da população com mais de 18 anos está acima do peso e 20,8\% são obesos e esses valores vem crescendo. De 2006 a 2015, o número de pessoas com sobrepeso aumentou 60,1\% e com obesidade aumentou $26,5 \%$. No público infantil o perfil de crescimento não é diferente, das crianças analisadas no período de 2008 a 2013 nas faixas de 0 a 5 anos o crescimento na porcentagem de obesidade foi de 79\%, de acordo com dados da Fundação Abrinq. Ainda não existem trabalhos que abordem obesidade e dislipidemia em crianças residentes na cidade de Goiânia. Diante disso, observou-se a necessidade de desenvolver uma pesquisa que tem como objetivo investigar a presença de dislipidemias e o estado nutricional em um grupo de crianças e adolescentes, de zero a doze anos, residentes no município de Goiânia.

\section{Metodologia}

Foi realizado um estudo analítico, do tipo série de casos, na qual foram analisados 130 prontuários eletrônicos de pacientes de 0 a 12 anos. Dos 130 prontuários, 75 eram de pacientes usuários do CAIS Jardim América atendidos por uma nutricionista, e 65 de pacientes usuários da clínica Centro Análise, atendidos por um pediatra em Goiânia-Goiás. Os prontuários foram analisados no mês de julho de 2017. Os critérios usados para escolha dos prontuários foram: idade entre $0 \mathrm{e}$ 12 anos, ser residente em Goiânia e ter os dados de peso, altura e bairro em que reside preenchidos no prontuário. Foram analisados dez prontuários de cada idade.

Os dados retirados dos prontuários para a pesquisa proposta foram: gênero, idade, altura, peso, bairro e exames referentes ao perfil lipídico quando presentes no prontuário. O Score Z é uma medida utilizada para determinar o estado 
nutricional de um paciente, ela é padronizada pela OMS, é mais especifica e a mais utilizada pelos nutricionistas e consegue ser mais precisa no risco nutricional, além de auxiliar a análise do desenvolvimento infanto-juvenil. O Score Z das crianças e adolescentes foi calculado no software profissional Cookie ${ }^{\circledR}$, programa criado seguindo os critérios do SISVAN/DATASUS que foi fundamentado pelos parâmetros determinados pela OMS. O percentil é uma medida também sensível, porém não estima uma situação de risco. A Tabela 1, a seguir, mostra como estas medidas estabelecem o estado nutricional:

Tabela 1: Índices antropométricos.

\begin{tabular}{|c|c|c|c|c|c|c|c|c|}
\hline \multirow{3}{*}{\multicolumn{2}{|c|}{ VALORES CRIIICOS }} & \multicolumn{7}{|c|}{ INDICES ANTROPOMÉTRICOS } \\
\hline & & \multicolumn{4}{|c|}{ CFIANCAS DE D A 5 ANOS INCOMPIEIOS } & \multicolumn{3}{|c|}{$\begin{array}{l}\text { CRIANCAS DE } 5 \text { A } 10 \text { ANOS } \\
\text { INCOMPUEIOS }\end{array}$} \\
\hline & & $\begin{array}{l}\text { Peso para } \\
\text { idade. }\end{array}$ & $\begin{array}{l}\text { Peso pare: } \\
\text { estature }\end{array}$ & $\begin{array}{l}\text { MC parn } \\
\text { idade }\end{array}$ & $\begin{array}{l}\text { Estatura } \\
\text { para } \\
\text { ldade }\end{array}$ & $\begin{array}{l}\text { Pero para } \\
\text { idade }\end{array}$ & $\begin{array}{l}\text { IMCpara: } \\
\text { Idade }\end{array}$ & $\begin{array}{l}\text { Estatura } \\
\text { para } \\
\text { idade }\end{array}$ \\
\hline $\begin{array}{c}\text { < Percentil } \\
0,1\end{array}$ & $\begin{array}{c}<\text { Escore- } 2 \\
-3\end{array}$ & $\begin{array}{c}\text { Muito } \\
\text { baixo peso } \\
\text { para a } \\
\text { idade }\end{array}$ & $\begin{array}{l}\text { Magreza } \\
\text { acentuada }\end{array}$ & $\begin{array}{l}\text { Magreza } \\
\text { acentuada }\end{array}$ & $\begin{array}{c}\text { Muito } \\
\text { baixa } \\
\text { estatura } \\
\text { para a } \\
\text { idade }\end{array}$ & $\begin{array}{c}\text { Muito } \\
\text { baixo peso } \\
\text { para a } \\
\text { idade }\end{array}$ & $\begin{array}{l}\text { Magreza } \\
\text { acentuada }\end{array}$ & $\begin{array}{c}\text { Muito } \\
\text { baixa } \\
\text { estatura } \\
\text { para a } \\
\text { idade }\end{array}$ \\
\hline $\begin{array}{c}\geq \text { Percentil } \\
0,1 \mathrm{e}< \\
\text { Percentil } 3\end{array}$ & $\begin{array}{c}\geq \text { Escore-z } \\
-3 \mathrm{e}< \\
\text { Escore-z-2 }\end{array}$ & $\begin{array}{l}\text { Baixo } \\
\text { peso para } \\
\text { a idade }\end{array}$ & Magreza & Magreza & $\begin{array}{c}\text { Baixa } \\
\text { estatura } \\
\text { para a } \\
\text { idade }\end{array}$ & $\begin{array}{c}\text { Baixo peso } \\
\text { para a } \\
\text { idade }\end{array}$ & Magreza & $\begin{array}{c}\text { Baixa } \\
\text { estatura } \\
\text { para a } \\
\text { idade }\end{array}$ \\
\hline $\begin{array}{c}\geq \text { Percentil } \\
3 \mathrm{e}< \\
\text { Percentil } \\
15\end{array}$ & $\begin{array}{c}\geq \text { Escore-z } \\
-2 \mathrm{e}< \\
\text { Escore-z-1 }\end{array}$ & \multirow{3}{*}{$\begin{array}{c}\text { Peso } \\
\text { adequado } \\
\text { para a } \\
\text { idade }\end{array}$} & \multirow{2}{*}{ Eutrofia } & \multirow{2}{*}{ Eutrofia } & \multirow{5}{*}{$\begin{array}{c}\text { Estatura } \\
\text { adequada } \\
\text { para a } \\
\text { idade }^{2}\end{array}$} & \multirow{3}{*}{$\begin{array}{c}\text { Peso } \\
\text { adequado } \\
\text { para a } \\
\text { idade }\end{array}$} & \multirow{2}{*}{ Eutrofia } & \multirow{5}{*}{$\begin{array}{c}\text { Estatura } \\
\text { adequada } \\
\text { para a } \\
\text { idade }{ }^{2}\end{array}$} \\
\hline $\begin{array}{c}\geq \text { Percentil } \\
15 \text { e } \leq \\
\text { Percentil } \\
85\end{array}$ & $\begin{array}{c}\geq \text { Escore-z } \\
-1 \text { e } \leq \\
\text { Escore-z+1 }\end{array}$ & & & & & & & \\
\hline $\begin{array}{c}>\text { Percentil } \\
85 \text { e } \leq \\
\text { Percentil } \\
97\end{array}$ & $\begin{array}{c}>\text { Escore-z } \\
+1 \text { e } \leq \\
\text { Escore-z+2 }\end{array}$ & & $\begin{array}{l}\text { Risco de } \\
\text { sobrepeso }\end{array}$ & $\begin{array}{l}\text { Risco de } \\
\text { sobrepeso }\end{array}$ & & & Sobrepeso & \\
\hline $\begin{array}{c}>\text { Percentil } \\
97 \text { es } \\
\text { Percentil } \\
99,9\end{array}$ & $\begin{array}{c}>\text { Escore-z } \\
+2 \mathrm{e} \leq \\
\text { Escore-z+3 }\end{array}$ & \multirow{2}{*}{$\begin{array}{c}\text { Peso } \\
\text { elevado } \\
\text { para a } \\
\text { idade }^{1}\end{array}$} & Sobrepeso & Sobrepeso & & \multirow{2}{*}{$\begin{array}{c}\text { Peso } \\
\text { elevado } \\
\text { para a } \\
\text { idade }^{2}\end{array}$} & Obesidade & \\
\hline $\begin{array}{c}>\text { Percentil } \\
99,9\end{array}$ & $\begin{array}{c}>\text { Escore-z } \\
+3\end{array}$ & & Obesidade & Obesidade & & & $\begin{array}{l}\text { Obesidade } \\
\text { grave }\end{array}$ & \\
\hline
\end{tabular}

Fonte: Adaptado de Organización Mundial de la Salud. Curso de Capacitación sobre la evaluación del crecimiento del niño. Versión 1 - Noviembre 2006. Ginebra, OMS, 2006.

O Score Z foi utilizado para classificar as crianças e adolescentes de acordo com seu estado nutricional: obesidade grave, obesidade, sobrepeso, eutrofia, magreza e magreza acentuada (Melo et al., 2011). Já a classificação para as dislipidemias foi feita a partir de alterações nas dosagens de colesterol-total, triglicerídeo, colesterol-HDL, colesterol-LDL e/ou colesterol-VLDL. Para análise dos dados as amostras foram divididas em 4 grupos: 0 a 3 anos, 4 a 6 anos, 7 a 9 anos e 10 a 12 anos (Melo et al., 2011).

O sigilo das informações coletadas será mantido, somente os autores da pesquisa tiveram acesso aos dados, que foram coletados diretamente nas unidades de saúde em sala própria e com privacidade garantida, nenhuma imagem foi feita dos prontuários, o que corrobora a confidencialidade dos dados. Os dados obtidos foram dispostos em planilhas no Microsoft Excel® (versão Office 2007) e para o processamento dos resultados utilizou-se estatística descritiva.

O procedimento foi submetido e aprovado pelo Comitê de Ética em Pesquisa com seres Humanos (CEP) da Pontifícia Universidade Católica de Goiás (PUC Goiás), dessa forma o trabalho de número de parecer: 2.100.562 está de acordo com a Conselho Nacional de Saúde (CNS) 196/96, que diz que todo trabalho envolvendo humanos direta ou indiretamente pode causar danos atuais ou tardios aos participantes. Neste caso, é um estudo indireto com seres humanos, que não causam danos, 
tanto no presente quanto no futuro, já que nosso único contato com eles foi através de fichas e os nomes/identidades não foram e não serão revelados.

\section{Resultados}

Foram analisados 130 prontuários, de pacientes de 0 a 12 anos, residentes em Goiânia. Do gênero feminino foram coletados 68 prontuários $(52,3 \%)$, e do gênero masculino foram 62 prontuários $(47,7 \%)$. A análise foi dividida em quatro grupos de diferentes faixas etárias e foram analisados estado nutricional, dislipidemia e a associação de dislipidemia e obesidade em crianças e adolescentes. A divisão por faixa etária pode ser observada na Tabela 2.

Tabela 2: Distribuição dos grupos com relação a faixa etária.

\begin{tabular}{c|c|c|}
\hline Grupos & Número de participantes & Faixa Etária em anos \\
\hline Grupo 1 & 40 & zero a tres \\
\hline Grupo 2 & 30 & quatro a seis \\
\hline Grupo 3 & 30 & sete a nove \\
\hline Grupo 4 & 30 & dez a doze \\
\hline TOTAL & $\mathbf{1 3 0}$ & Zero a doze \\
\hline
\end{tabular}

Fonte: Dados da pesquisa.

No grupo 1, das 40 crianças de 0 a 3 anos analisadas, com relação ao estado nutricional, 2 apresentaram obesidade, 1 sobrepeso, 5 apresentaram risco de sobrepeso; 32 apresentaram eutrofia e nenhum apresentou magreza ou magreza acentuada. Neste grupo, $60 \%$ eram do gênero feminino e $40 \%$ do gênero masculino.

Tabela 3: Estado Nutricional do grupo de crianças de 0 a 3 anos.

\begin{tabular}{|c|c|c|}
\hline Estado Nutricional & Número de crianças & Porcentagem \\
\hline Obesidade & 2 & $5 \%$ \\
\hline Sobrepeso & 1 & $3 \%$ \\
\hline Risco de Sobrepeso & 5 & $12 \%$ \\
\hline Eutrofia & 32 & $80 \%$ \\
Magreza & 0 & $0 \%$ \\
\hline Magreza Acentuada & 0 & $0 \%$ \\
\hline TOTAL & $\mathbf{4 0}$ & $\mathbf{1 0 0} \%$ \\
\hline
\end{tabular}

Fonte: Dados da pesquisa.

Em relação as dosagens laboratoriais avaliadas foram identificadas 3crianças com aumento no colesterol total, sendo 1 do gênero feminino e 2 do gênero masculino; em relação ao triglicerídeo, 1 criança do gênero masculino apresentou elevação. Com os dados laboratoriais apresentados observou-se que 4 crianças apresentaram dislipidemia, ou seja 13,3\% do grupo de 0 a 3 anos. 
Gráfico 1: Alterações laboratoriais referentes ao perfil lipídico coletadas dos prontuários de crianças de 0 a 3 anos.

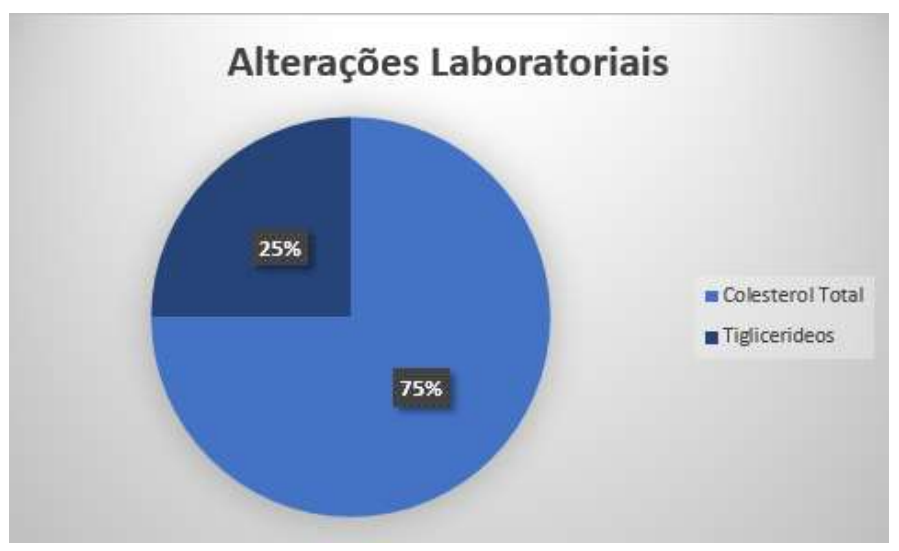

Fonte: Dados da pesquisa.

A associação entre obesos e dislipemicos no grupo explorado foi de 0\%. No segundo grupo, de 4 a 6 anos foram avaliadas 30 crianças. Em relação ao estado nutricional observou-se que: uma apresentou obesidade grave, 5obesidade, 3 apresentavam sobrepeso, 4 com risco de sobrepeso, 16 eutróficos, uma magreza e nenhum tinha magreza acentuada. Neste grupo $53,3 \%$ eram do gênero feminino e $46,7 \%$ do gênero masculino.

Tabela 4: Estado Nutricional do grupo de crianças de 4 a 6 anos.

\begin{tabular}{|c|c|c|}
\hline Estado Nutricional & Número de crianças Porcentagem \\
\hline *Obesidade Grave & 1 & $3 \%$ \\
\hline Obesidade & 5 & $17 \%$ \\
\hline Sobrepeso & 3 & $10 \%$ \\
\hline Risco de Sobrepeso & 4 & $13 \%$ \\
\hline Eutrofia & 16 & $54 \%$ \\
\hline Magreza & 1 & $3 \%$ \\
\hline Magreza Acentuada & 0 & $0 \%$ \\
\hline TOTAL & $\mathbf{3 0}$ & $\mathbf{1 0 0} \%$ \\
\hline
\end{tabular}

${ }^{*}$ Crianças até 5 anos não são classificadas com obesidade grave de acordo com a OMS, dessa forma, todo o percentual de obesos graves são referentes a crianças de 6 anos. Fonte: Dados da pesquisa.

Neste grupo foram encontradas 6 crianças com aumento nas dosagens de colesterol total, sendo 4 do gênero feminino e 2 do gênero masculino, 3 com elevação em triglicerídeo, sendo todas do gênero feminino. A partir destes dados observou-se que $30 \%$ das crianças apresentavam dislipidemia. 
Gráfico 2: Alterações laboratoriais referentes ao perfil lipídico coletadas dos prontuários de crianças de 4 a 6 anos.

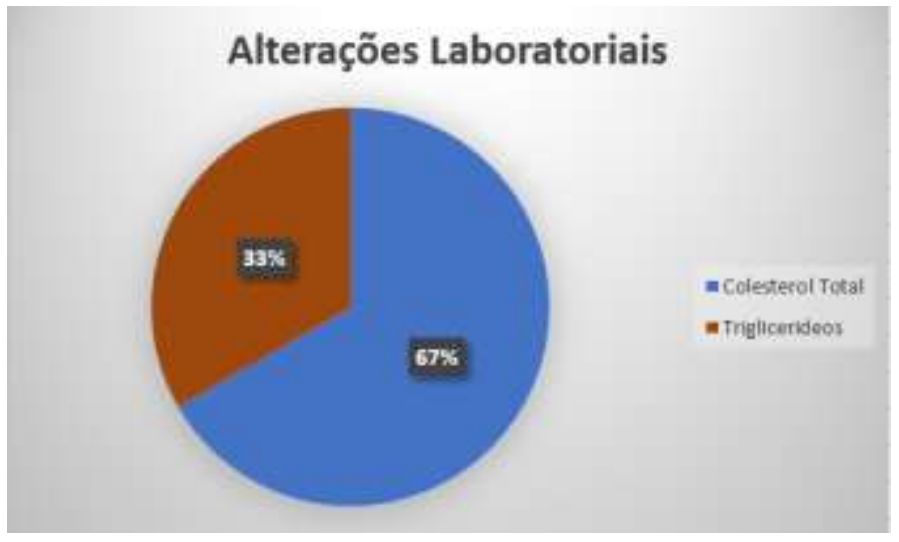

Fonte: Dados da pesquisa.

A associação entra obesos e dislipêmicos neste grupo foi de 6,7\%. No terceiro grupo, 7 a 9 anos, 30 crianças foram avaliadas. Os resultados encontrados foram marcantes: 7 com obesidade grave, 7 com obesidade, 3 com sobrepeso, 13 eutróficos, e nenhum com magreza acentuada ou magreza. Neste grupo, 43,3\% eram do sexo feminino e 56,7\% do sexo masculino.

Tabela 5: Estado Nutricional do grupo de crianças de 7 a 9 anos.

\begin{tabular}{|c|c|c|}
\hline Estado Nutricional & Número de crianças & Porcentagem \\
\hline Obesidade Grave & 7 & $23 \%$ \\
\hline Obesidade & 7 & $23 \%$ \\
\hline Sobrepeso & 3 & $10 \%$ \\
\hline Eutrofia & 13 & $44 \%$ \\
\hline Magreza & 0 & $0 \%$ \\
\hline Magreza Acentuada & 0 & $0 \%$ \\
\hline TOTAL & $\mathbf{3 0}$ & $\mathbf{1 0 0} \%$ \\
\hline
\end{tabular}

Fonte: Dados da pesquisa.

No grupo de 7 a 9 anos foram encontradas as seguintes alterações laboratoriais: 10 crianças com elevação no exame de colesterol total, sendo 4 do gênero feminino e 6 do gênero masculino, 4 com alteração em triglicerídeo, sendo 2 do gênero feminino e 2 do gênero masculino, 2 com elevação em LDL, ambas do gênero masculino, 1 com elevação de VLDL e redução de HDL, todos do gênero masculino. Sendo assim, 56,7\% apresentavam dislipidemia.

Gráfico 3: Alterações laboratoriais referentes ao perfil lipídico coletadas dos prontuários de crianças de 7 a 9 anos.

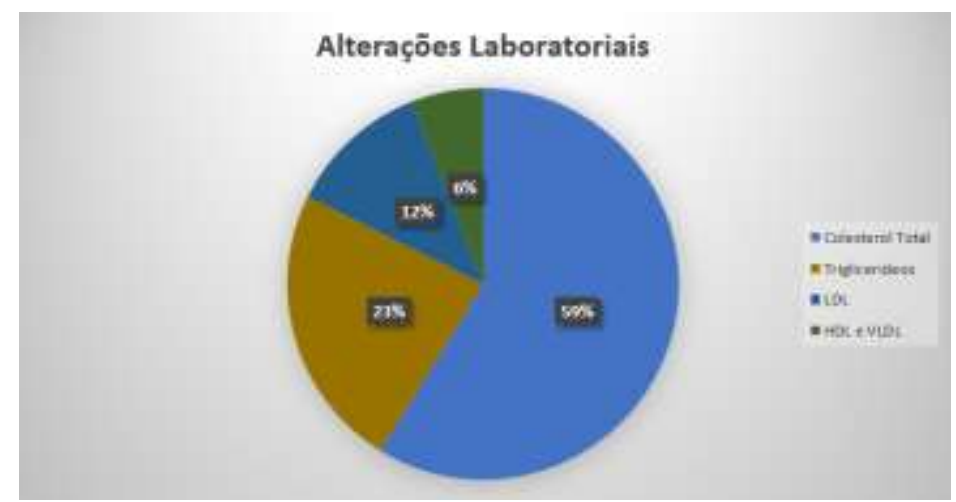

Fonte: Dados da pesquisa. 
A associação entre obesos e dislipêmicos neste grupo foi de 6,7\%. E por fim, os 30 adolescentes de 10 a 12 anos que também obtiveram resultados importantes para a pesquisa: 3com obesidade grave, 18 obesos, 1 com sobrepeso, 8 eutrófico sem nenhum com magreza ou magreza acentuada. Neste grupo, 50\% eram do gênero feminino e 50\% do gênero masculino.

Tabela 6: Estado Nutricional do grupo de adolescentes de 10 a 12 anos.

\begin{tabular}{|c|c|c|}
\hline Estado Nutricional & Número de crianças Porcentagem \\
\hline Obesidade Grave & 3 & $10 \%$ \\
\hline Obesidade & 18 & $60 \%$ \\
\hline Sobrepeso & 1 & $3 \%$ \\
\hline Eutrofia & 8 & $27 \%$ \\
\hline Magreza & 0 & $0 \%$ \\
\hline Magreza Acentuada & 0 & $0 \%$ \\
\hline TOTAL & $\mathbf{3 0}$ & $\mathbf{1 0 0} \%$ \\
\hline
\end{tabular}

Fonte: Dados da pesquisa.

No total, 6 adolescentes apresentaram elevação no exame de colesterol total sendo 3 do gênero feminino e 3 do gênero masculino, 5 apresentaram alteração na dosagem de triglicerídeo sendo 2 do gênero feminino e 3 do gênero masculino. Assim, $36,7 \%$ apresentaram dislipidemia.

Gráfico 4: Alterações laboratoriais referentes ao perfil lipídico coletadas dos prontuários de adolescentes de 10 a 12 anos.

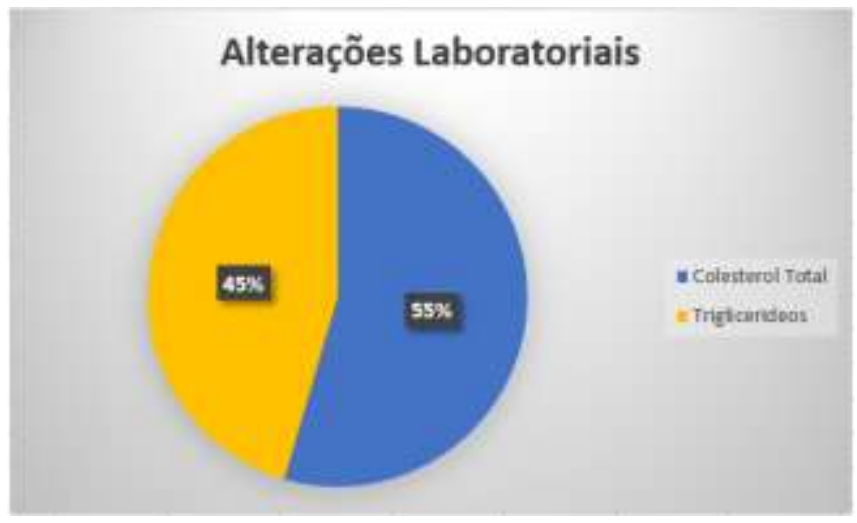

Fonte: Dados da pesquisa.

A associação entre obesos e dislipemicos neste grupo foi de 13,3\%. Com estes resultados pode-se afirmar que das 130 crianças e adolescentes analisadas $6,1 \%$ apresentavam sobrepeso, 24,7\% eram obesas, $8,46 \%$ apresentavam obesidade grave, $53 \%$ eutróficos, $0,8 \%$ apresentavam magreza, 6,9\% mostraram risco de sobrepeso e nenhuma criança apresentou magreza acentuada. A porcentagem de dislipidemia nas crianças e adolescentes foi de $23 \%$, nas crianças de 0 a 9 anos foi encontrado percentual de $21 \%$ e nos adolescentes de 10 a 12 anos de $36,7 \%$

\section{Discussão}

O processo de urbanização e o desenvolvimento econômico afetaram o estilo de vida da população e dessa forma o sedentarismo aumentou e os padrões de alimentação tornaram-se cada vez mais inadequados (Oliveira et al., 2003). Dados fornecidos pela Fundação Abrinq no ano de 2015 mostram que o número de crianças obesas de 0 a 5 cinco anos cresceu 79\% entre os anos de 2008 a 2013. 
Os dados encontrados neste presente estudo são preocupantes. O quantitativo de adolescentes de 10 a 12 anos com obesidade foi de $60 \%$, um valor muito alto, que pode ser explicado pela fase em que esta população se encontra, o indivíduo sofre grandes mudanças biopsicossociais na qual seu organismo está em fase de desenvolvimento e precisa de uma demanda energética maior, além da etnia e condições econômicas que determinam a alimentação deste indivíduo. $\mathrm{O}$ consumo de alimentos ricos em açucares, gorduras e a falta da ingestão de verduras, frutas e hortaliças tem agravado esses casos (Brasil, 2014).

Outro fator inquietante foi o das crianças em fase pré-escolar (dois aos seis anos) com níveis de obesidade grave de $4 \%$ (exclusivo para crianças acima de 6 anos), de obesidade de 12\%, de sobrepeso de $6 \%$ e risco de sobrepeso de 14\%. Isso pode ser justificado pelo desmame precoce e substituído pelas formulas, o período gestacional o estilo de vida dos familiares, além disso pré-natal inadequado incluindo o sedentarismo (Gama et al., 2007; Brasil, 2014).

Estudo publicado em 2004, após avaliar 231 crianças, observou que 68,4\% delas apresentavam dislipidemia de qualquer natureza, colesterol total ou frações e desses 18,6\% tinham alteração de LDL-colesterol, 35\% aumento de HDL e 3,5\% de triglicérides (Gama et al., 2007).

Os resultados encontrados neste estudo são divergentes com os dados publicados por Moura et al. (2000), feito em Campinas com 1.600 escolares de 7 a 14 anos onde foi encontrado um percentual de 35\% de dislipidemia entre crianças e adolescentes. No presente estudo foram encontrados $23 \%$ de dislipidemia no público de 130 indivíduos de 0 a 12 anos. A divergência dos resultados pode ter acontecido pelo número maior de crianças e adolescentes analisados no estudo de Moura et al. (2000), além da faixa etária mais elevada. Observou também um número de meninas com elevação de triglicerídeos e colesterol total foi maior do que nos meninos. Este quadro não vai de encontro com o estudo nas crianças de sete a nove anos deste trabalho, a relação de dislipidemia foi igual para ambos os gêneros.

Já o estudo feito por Guiliano et al. no município de Florianópolis, em 2005, foi o que mais se aproximou dos resultados desta pesquisa em Goiânia. Observaram um percentual de 22\% de dislipêmicos após avaliarem1.053 crianças e adolescentes de 7 a 15 anos de idade. Dados muito próximos do encontrado no percentual de dislipidemia das 130 crianças e adolescentes de 0 a 12 anos de Goiânia foi de 23\% (Giuliano et al., 2005).

Comparando com outro estudo sobre dislipidemia, em Salvador, Neto et al. (2012) mostrou um percentual de 20,4\% das 1.131 crianças e adolesceste de 7 a 14 anos apresentavam dislipidemia. O estudo realizado em Goiânia apresentou valores semelhantes possivelmente por ser um estudo mais recente.

Trabalho realizado em Londrina-PR, em que foram encontrados 20,8\% de dislipêmicos do total de 2.029 crianças e adolescentes de 7 a 17 anos (Seki et al., 2007) também apresentam valores parecidos com o de Goiânia.

As com menor percentual foram as encontradas em Bento Gonçalves-RS das 1.501 criança e adolescentes de 3 a 15 anos, somente 10,33\% apresentavam dislipidemia, em 1997 (Gerber et al., 1997). Este estudo também se difere do presente estudo de Goiânia que apresentou 23\%, provavelmente pelo número de indivíduos analisados. Em Itajaí-SC com 257 crianças e adolescentes de 3 a 14 anos o percentual foi de apenas 4,7\% no ano de 2005 (Grillo et al., 2005).O presente estudo mostra um percentual de dislipidemia de $23 \%$.

Estudos feitos pelo IBGE (2003), entre 2002 a 2003, comprovam que a fase do início da adolescência e final da infância, é a fase que apresenta maior número de obesidade e sobrepeso. Das faixas de 10 a 11 anos o quantitativo de sobrepeso foi de $17,7 \%$ e de obesidade foi 3,7\%. De 12 a 14 anos o valor encontrado para sobrepeso foi 14,3\% e obesidade foi 2,4\% (Inácio et al., 2003). Os presentes resultados desta pesquisa apresentam informações de obesidade e sobrepeso divergentes, no qual os adolescentes de 10 a 12 anos apresentaram um percentual de sobrepeso de $3 \%$ e de obesidade de $60 \%$ (Gama et al., 2007). 
Um trabalho realizado no Pernambuco mostrou que 29,7\% de 414 crianças e adolescentes apresentavam dislipidemias, 4\% tinham sobrepeso e as do gênero feminino apresentavam triglicerídeos e colesterol total maior do que as do gênero masculino, e não foram encontradas divergências nos valores dos exames de colesterol e triglicerídeos entre crianças e adolescentes, o que é bem preocupante, já que estão em fase de desenvolvimento (De Franca et al., 2006). Os resultados da presente pesquisa indicam que das 130 crianças analisadas, 11,5\% apresentaram sobrepeso e 23\% dislipidemia. Já em relação ao gênero e aos níveis de colesterol total e triglicerídeos, as do gênero masculino apresentaram percentual de colesterol total maior que as do gênero feminino,35,1\% e 32,3\%, respectivamente. Em relação aos triglicerídeos o percentual foi de 16,2\% para ambos os gêneros.

De acordo com estudo feito por Cook e Kavey em 2011, o mais comum entre as crianças é o aumento isolado de colesterol total e com o presente estudo em Goiânia não foi diferente, cerca de 12,3\% do total apresentam elevação apenas de colesterol total, cerca de 53\% do total das dislipidemias (Cook \& Kavey, 2011).

\section{Conclusão}

Podemos considerar que crianças e adolescentes da região da metropolitana de Goiânia apresentam um indíce de obesidade e dislipidemia muito elevado apresentado pelo nosso estudo. Isso pode prejudicar tanto as atividades delas como criança, por exemplo, cansaço precoce ao praticar atividades físicas, como pode também desencadear problemas sociais como o bullying (violência física e psicológica). Além disso pode prejudicar a saúde na fase adulta, gerando problemas cardiovasculares e neurovasculares, síndrome metabólica, diabetes. Esta é uma doença estigmatizante e deve ser diagnosticada e tratada da forma precoce para evitar preconceitos, medicações, outras doenças e síndromes associadas, procedimento invasivos e até cirurgias como: bariátrica, redução do estomago e outras.

Os níveis de gordura no sangue das crianças podem não ser derivados apenas da má alimentação, fatores genéticos são determinantes nessa faixa etária (o que possivelmente pode explicar a relação de $0 \%$ de obesidade e dislipidemia nas crianças de 0 a 3 anos) e o acompanhamento dos pais e responsáveis é muito importante para que tenha um diagnóstico precoce, já que essa é a melhor alternativa para que essas crianças tenham uma vida adulta mais saudável e longa, o acompanhamento com um médico, de um nutricionista e a pratica de atividades físicas regulares é imprescindível.

Esse estudo traz informações que vão ajudar não só os pais e responsáveis a analisarem e repensarem os costumes e rotinas de suas crianças, como os adultos, os futuros pais, os profissionais da saúde, as professoras das escolas tanto particulares quanto públicas a observar melhor as crianças e seus comportamentos. A prefeitura da cidade também pode tomar medidas preventivas ou tratamentos para a população infanto-juvenil da cidade, desde a conscientização da doença e seus riscos, na promoção de atividades ao ar livre e no fornecimento constante dos medicamentos necessários, a contratação de mais profissionais da saúde para que a população possa ser assistida.

\section{Referências}

Avezum, J. A., Feldamn, A., Carvalho, AC de C., Sousa, A. C. S., Mansur A. P., Bozza A. E. Z., et al. (2015) V Diretriz da Sociedade Brasileira de Cardiologia sobre Tratamento do Infarto Agudo do Miocárdio com Supradesnível do Segmento ST. Arq Bras Cardio, 105(2), 1-121.

Brasil. Inquérito Domiciliar sobre Comportamentos de Risco e Morbidade Referida de Doenças e Agravos Não Transmissíveis. (2004). Ministério da Saúde Secr Vigilância em Saúde Secr Atenção à Saúde Inst Nac Câncer Coord Prevenção e Vigilância, 1-184.

Brasil. Ministério da Saúde. Protocolos do Sistema de Vigilância Alimentar e Nutricional - SISVAN na assistência à saúde. (2008). Série B. Textos Básicos de saúde).

Brasil - Rede Nacional Primeira Infância. (2014). Obesidade infantil - Projeto Observatório Nacional da Primeira Infância. Mapeamento da Ação Finalística Criança com Saúde.

Cook, S., \& Kavey, R. E. W. Dyslipidemia and pediatric obesity. (2011). Pediatric Clinics of North America., $102(3), 1363-1373$. 
Research, Society and Development, v. 11, n. 1, e25711124671, 2022

(CC BY 4.0) | ISSN 2525-3409 | DOI: http://dx.doi.org/10.33448/rsd-v11i1.24671

De Franca E., \& Alves, J. G. B. (2006). Dyslipidemia among adolescents and children from Pernambuco. Arq Bras Cardiol, 87(6):722-727.

Dos Anjos L. A. (2006). Agravos à saúde e epidemiologia da obesidade. Obesidade e saúde pública 29-39.

Enes, C. C. (2010).Obesity in adolescence and its main determinants. Revista Brasileira de Epidemiologia, 13(1), 163-71.

Fernandes, R. A., Christofaro, D. G., Casonatto, F., Codogno, J. S., Rodrigues, E. Q., Cardoso, M. L. et al. (2011). Prevalence of dyslipidemia in individuals physically active during childhood, adolescence and adult age. Arq Bras Cardiol, 97:317-323.

Gama, S. R., Carvalho, M. S., \& Chaves, C. R. M. (2007). Prevalência em crianças de fatores de risco para as doenças cardiovasculares. Cad Saude Publica, 23(9): 2239-45

Gerber, Z. R., \& Zielinsky P. (1997). Risk factors for atherosclerosis in children: an epidemiologic study. Arq Bras Cardiol, 69(4):231-236.

Grillo, L. P., Crispim, S. P., Rossi, A., \& Campos, I. C. (2005). Perfil lipídico e obesidade em escolares de baixa renda. Rev Bras Epidemiol, 8(1): 75-81

Giuliano, I. C. B., Coutinho, M. S. S., Freitas, S. F.T., Pires, M. M., Zunino, J. N., \& Ribeiro, R. Q. C. (2005). Serum Lipids in School Kids and Adolescents from Florianópolis , SC, Brazil . Arq Bras Cardiol, 85(2):1-6.

Hafe, P. V. (2015). Dislipidemia e obesidade. Rev Fatores Risco, 38:92-97.

Inácio, L., Ibge, E., Côrtes, C., \& Tai, D. W. (2003). Orçamentos Familiares 2002-2003: Análise da disponibilidade domiciliar de alimentos e do estado nutrional no Brasil. American Journal of Cardiology, 46(2): 983-987.

Lima, N. P., Horta, B. L., Motta, J. V. S., Valença, M. S., Oliveira, V., \& Santos, T. V. (2015). Evolução do excesso de peso e obesidade até a idade adulta, Pelotas, Rio Grande do Sul, Brasil, 1982-2012. Cad Saude Publica ,31(9): 2017-2025.

Melo, M. E. (2011), Doenças Desencadeadas ou Agravadas pela Obesidade. Abeso, 4(1): 8-15.

Mello, E. D., Luft, V. C., \& Meyer F. (2004). Childhood obesity - Towards effectiveness. J Pediatr (Rio J), 80(3):173-82.

Moura, E. C., De Castro, C. M., Mellin, A. S., \& De Figueiredo, D. B. (2000). Perfil lipídico em escolares de Campinas, SP, Brasil. Rev Saude Publica, 34(5): 499-505.

Neto, O. D. A., Silva, R. C. R., Assis, A. M. O., \& Pinto, E. J. (2012). Fatores associados à dislipidemia em crianças e adolescentes de escolas públicas de Salvador, Bahia. Rev Bras Epidemiol, 15(2): 335-45.

Oliveira, C. L., \& Fisberg, M. (2003). Obesidade na Infância e Adolescência - Uma verdadeira Epidemia. Arq Bras Endocrinol Metabol, 47(2):107-108.

Santos, J. E., Guimarães, A. C., \& Diament, J. (1999). Consenso Brasileiro Sobre Dislipidemias Detecção, Avaliação e Tratamento. Arq Bras Endocrinol Metabol, 43(4): 287-305.

Seki, M. O., Matsuo, T., \& Seki, M. (2007). Colesterol não-HDL em escolares de 7 a 17 anos de idade em um município brasileiro. Rev Panam Saúde Pública, 21(5): 307-12. 